\title{
Dependence of the critical temperature on microscopic parameters in the three-dimensional Ising-like systems
}

\author{
M.P.Kozlovskii, V.V.Dukhovii, I.V.Pylyuk \\ Institute for Condensed Matter Physics \\ of the National Academy of Sciences of Ukraine, \\ 1 Svientsitskii Str., 79011 Lviv, Ukraine
}

Received March 21, 2000, in final form November 5, 2000

The critical behaviour of the three-dimensional Ising-like system on a simple cubic lattice with an exponentially decreasing interaction potential is investigated within the collective variables method. The solution of the equation for the phase transition temperature is obtained by taking into account the dependence of its coefficients on the temperature. The dependence of the critical temperature on the interaction potential range is studied.

Key words: Ising-like systems, critical behaviour, phase transitions, collective variables, phase transition temperature

PACS: $05.50 .+q, 64.60 . F r, 75.10 . H k$

This paper gives rise into the theory of second order phase transitions in the approach developed by I.R.Yukhnovskii [1]. In a number of recent papers [2-5], the explicit expressions for basic thermodynamic functions of the three-dimensional Ising-like model have been obtained. In particular, the dependence of these functions on $\tau\left(\tau=\left(T-T_{\mathrm{c}}\right) / T_{\mathrm{c}}, T_{\mathrm{c}}\right.$ is the phase transition temperature), as well as on microscopic parameters of the model, was studied. On the other hand, the dependence of $T_{\mathrm{c}}$ itself on microscopic parameters has not been studied deeply enough so far. Such a study is our aim in this research.

We investigate a system of Ising spins located at the sites of a simple cubic lattice $\mathbb{L}:$

$$
\mathbb{L}=c \mathbb{Z}^{3}=\left\{\mathbf{l}=\left(l_{x}, l_{y}, l_{z}\right) \mid l_{i}=c \kappa_{i} ; \kappa_{i} \in \mathbb{Z}, i=x, y, z\right\} .
$$

The formal Hamiltonian of the system is

$$
H=-\frac{1}{2} \sum_{\mathbf{i} \mathbf{j}} \Phi\left(r_{\mathbf{i j}}\right) \sigma_{\mathbf{i}} \sigma_{\mathbf{j}}-h \sum_{\mathbf{i}} \sigma_{\mathbf{i}} .
$$

Here $h$ is an external field, $r_{\mathbf{i j}}$ is the distance between the particles at the sites $\mathbf{i}$ and $\mathbf{j}, \sigma_{\mathbf{i}}$ is an operator of the $z$-component of a spin in the $\mathbf{i}$-th site. The interaction 
potential $\Phi\left(r_{\mathbf{i j}}\right)$ is

$$
\Phi\left(r_{\mathrm{ij}}\right)=A \exp \left(-\frac{r_{\mathrm{ij}}}{b}\right), \quad A>0,
$$

where $b$ is the effective interaction range. In a general form its Fourier transform

$$
\tilde{\Phi}(k)=\sum_{\mathbf{i} \mathbf{j} \in \mathbb{L}} \Phi\left(r_{\mathbf{i j}}\right) \exp \left(i \mathbf{k r}_{\mathbf{i j}}\right), \quad \mathbf{k}=\left(k_{x}, k_{y}, k_{z}\right), \quad k_{i} \in\left(-\frac{\pi}{c}, \frac{\pi}{c}\right), \quad i=x, y, z
$$

is determined as

$$
\tilde{\Phi}(k)=\frac{\tilde{\Phi}(0)}{\left(1+b^{2} \mathbf{k}^{2}\right)^{2}}, \quad \tilde{\Phi}(0)=8 \pi A(b / c)^{3}, \quad k=|\mathbf{k}| .
$$

Let us consider our model taken in a box

$$
\Lambda=\left\{\mathbf{l}=\left(l_{x}, l_{y}, l_{z}\right) \mid l_{i}=c \kappa_{i} ; \kappa_{i}=1,2, \ldots, N_{i}, i=x, y, z\right\} ; \quad|\Lambda|:=N=N_{x} N_{y} N_{z}
$$

with periodic conditions on its boundaries. Accordingly, the set of values of $\mathbf{k}$ (Brillouin zone) now is

$$
\mathcal{B}=\left\{\mathbf{k}=\left(k_{x}, k_{y}, k_{z}\right) \mid k_{i}=-\frac{\pi}{c}+\frac{2 \pi}{c} \frac{\kappa_{i}}{N_{i}} ; \kappa_{i}=1,2, \ldots, N_{i}\right\} .
$$

We replace (3) by approximation

$$
\tilde{\Phi}(k)= \begin{cases}\tilde{\Phi}(0)\left(1-2 b^{2} \mathbf{k}^{2}\right), & \mathbf{k} \in \mathcal{B}^{\prime} \\ \tilde{\Phi}(0) \bar{\Phi}, & \mathbf{k} \in \mathcal{B} \backslash \mathcal{B}^{\prime}\end{cases}
$$

where the set

$$
\mathcal{B}^{\prime}=\left\{\mathbf{k}=\left(k_{x}, k_{y}, k_{z}\right) \mid k_{i}=-\frac{\pi}{c^{\prime}}+\frac{2 \pi}{c^{\prime}} \frac{\kappa_{i}^{\prime}}{N_{i}^{\prime}} ; \kappa_{i}^{\prime}=1,2, \ldots, N_{i}^{\prime}\right\}
$$

may serve as a Brillouin zone connected with a box

$$
\Lambda^{\prime}=\left\{\mathbf{l}=\left(l_{x}, l_{y}, l_{z}\right) \mid l_{i}=c^{\prime} \kappa_{i}^{\prime} ; \kappa_{i}^{\prime}=1,2, \ldots, N_{i}^{\prime}, i=x, y, z\right\}
$$

taken from the block lattice $\mathbb{L}^{\prime}=c^{\prime} \mathbb{Z}^{3}$ with $c^{\prime}=c s_{0}$ (see, for example, [1]). Here $N^{\prime}=N_{x}^{\prime} N_{y}^{\prime} N_{z}^{\prime}, N^{\prime}=N s_{0}^{-3}\left(s_{0}>1\right)$. The quantity $\bar{\Phi}$ in our calculations corresponds to the averaged value of $\tilde{\Phi}(k)$ given by $(3)$ over the set $\mathbf{k} \in \mathcal{B} \backslash \mathcal{B}^{\prime}[6]$. At $\tilde{\Phi}(0)=$ $2 d J, \quad b=b_{I}=c /(2 \sqrt{d})(J$ is the constant of the interaction between the nearest neighbours, $d$ is the space dimension) for small values of the wave vectors $\mathbf{k}$, the parabolic approximation of the Fourier transform of the exponentially decreasing interaction potential corresponds to the analogous approximation of the Fourier transform for the nearest neighbour interaction potential [7].

The critical behaviour of the three-dimensional Ising system is studied within the collective variables (CV) method $[1,8]$. This method permits to approximately calculate the partition function of the system and to obtain not only the universal 
quantities (critical exponents) but also analytic expressions for the thermodynamic functions in the vicinity of the phase transition point. The term CV is the collective name for a distinctive class of variables, which are specific for a given physical system. The CV-set includes the variables corresponding to the order parameters. Due to this, the CV phase space is natural for describing phase transitions. For magnetic systems, the CV are associated with the modes of the spin moment density oscillations. The $\mathrm{CV} \rho_{\mathbf{k}}^{c}, \rho_{\mathbf{k}}^{s}, \rho_{0}$ are determined using the following relations:

$$
\begin{aligned}
& \hat{\rho}_{\mathbf{k}}^{c}=\int \rho_{\mathbf{k}}^{c} \mathcal{J}(\rho-\hat{\rho})(\mathrm{d} \rho)^{N}, \\
& \hat{\rho}_{\mathbf{k}}^{s}=\int \rho_{\mathbf{k}}^{s} \mathcal{J}(\rho-\hat{\rho})(\mathrm{d} \rho)^{N}, \\
& \hat{\rho}_{0}=\int \rho_{0} \mathcal{J}(\rho-\hat{\rho})(\mathrm{d} \rho)^{N},
\end{aligned}
$$

where

$$
\mathcal{J}(\rho-\hat{\rho})=\delta\left(\rho_{0}-\hat{\rho}_{0}\right) \prod_{\mathbf{k}}^{\prime} \delta\left(\rho_{\mathbf{k}}^{c}-\hat{\rho}_{\mathbf{k}}^{c}\right) \delta\left(\rho_{\mathbf{k}}^{s}-\hat{\rho}_{\mathbf{k}}^{s}\right)
$$

is the transition operator,

$$
\begin{aligned}
& \hat{\rho}_{\mathbf{k}}^{c}=\frac{1}{\sqrt{N}} \sum_{\mathbf{j}} \sigma_{\mathbf{j}} \cos (\mathbf{k j}), \\
& \hat{\rho}_{\mathbf{k}}^{s}=\frac{1}{\sqrt{N}} \sum_{\mathbf{j}} \sigma_{\mathbf{j}} \sin (\mathbf{k} \mathbf{j}), \\
& \hat{\rho}_{0}=\frac{1}{\sqrt{N}} \sum_{\mathbf{j}} \sigma_{\mathbf{j}}
\end{aligned}
$$

are the spin density oscillation operators. The prime in (6) means that the product over $\mathbf{k}$ is performed in the upper semicube and that $k \neq 0,(\mathrm{~d} \rho)^{N}$ being the volume element:

$$
(\mathrm{d} \rho)^{N}=\mathrm{d} \rho_{0} \prod_{\mathbf{k}}^{\prime} \mathrm{d} \rho_{\mathbf{k}}^{c} \mathrm{~d} \rho_{\mathbf{k}}^{s} .
$$

For the sake of convenience, we introduce the variables

$$
\rho_{\mathbf{k}}=\rho_{\mathbf{k}}^{c}-\mathrm{i} \rho_{\mathbf{k}}^{s}
$$

which correspond to the operators

$$
\hat{\rho}_{\mathbf{k}}=\hat{\rho}_{\mathbf{k}}^{c}-\mathrm{i} \hat{\rho}_{\mathbf{k}}^{s}
$$

In this paper, the calculations are performed within the microscopic approach to the description of the critical behaviour of the three-dimensional Ising-like system [6]. The partition function of the system is evaluated using the non-Gaussian base measure density, which is represented as an exponential function of the CV the exponent of which involves the fourth order polynomial (the $\rho^{4}$ model). The functional 
representation for the partition function in the approximation of the $\rho^{4}$ model at $h=0$ reads

$$
\begin{aligned}
Z= & C^{\prime} e^{a_{0}^{\prime} N^{\prime}} \int \exp \left[-\frac{1}{2} \sum_{\mathbf{k} \in \mathcal{B}^{\prime}} d^{\prime}(k) \rho_{\mathbf{k}} \rho_{-\mathbf{k}}\right. \\
& \left.-\frac{a_{4}^{\prime}}{4 ! N^{\prime}} \sum_{\substack{\mathbf{k}_{1}, \ldots, \mathbf{k}_{4} \\
\mathbf{k}_{i} \in \mathcal{B}^{\prime}}} \rho_{\mathbf{k}_{1}} \cdots \rho_{\mathbf{k}_{4}} \delta_{\mathbf{k}_{1}+\cdots+\mathbf{k}_{4}}\right](\mathrm{d} \rho)^{N^{\prime}},
\end{aligned}
$$

where $C^{\prime}=2^{N} 2^{\left(N^{\prime}-1\right) / 2} \mathrm{e}^{\widetilde{\mathcal{M}_{0} N}}, \delta_{\mathbf{k}_{1}+\cdots+\mathbf{k}_{4}}$ is the Kronecker symbol. The coefficients in expression (10) are given by the relations (see [6])

$$
\begin{aligned}
& d^{\prime}(k)=a_{2}^{\prime}-\beta \tilde{\Phi}(k), \\
& a_{0}^{\prime}=\ln \left[(2 \pi)^{-1 / 2}\left(\frac{3}{\widetilde{\mathcal{M}}_{4}}\right)^{1 / 4} \exp \left(\frac{\tilde{y}^{2}}{4}\right) U(0, \tilde{y})\right] \\
& a_{2}^{\prime}=\left(\frac{3}{\widetilde{\mathcal{M}}_{4}}\right)^{1 / 2} U(\tilde{y}), \quad a_{4}^{\prime}=\frac{3}{\widetilde{\mathcal{M}}_{4}} \varphi(\tilde{y}), \\
& \tilde{y}=\sqrt{3} \widetilde{\mathcal{M}}_{2}\left(\widetilde{\mathcal{M}}_{4}\right)^{-1 / 2} .
\end{aligned}
$$

For the quantities $\widetilde{\mathcal{M}}_{2 l}$ we have

$$
\begin{aligned}
& \widetilde{\mathcal{M}}_{0}=\frac{1}{2} \alpha, \quad \widetilde{\mathcal{M}}_{2}=1-\alpha, \\
& \widetilde{\mathcal{M}}_{4}=2 s_{0}^{-3}(1-4 \alpha), \\
& \alpha=\beta \tilde{\Phi}(0) \bar{\Phi}\left(1-s_{0}^{-3}\right) .
\end{aligned}
$$

Here $\beta=1 /\left(k_{\mathrm{B}} T\right)$ is the inverse temperature, $k_{\mathrm{B}}$ is the Boltzmann constant. The special functions

$$
\begin{aligned}
U(\tilde{y}) & =\frac{U(1, \tilde{y})}{U(0, \tilde{y})} \\
\varphi(\tilde{y}) & =3 U^{2}(\tilde{y})+2 \tilde{y} U(\tilde{y})-2
\end{aligned}
$$

are combinations of the Weber parabolic cylinder functions

$$
U(a, t)=\frac{2}{\Gamma\left(a+\frac{1}{2}\right)} \mathrm{e}^{-t^{2} / 4} \int_{0}^{\infty} x^{2 a} \exp \left(-t x^{2}-\frac{1}{2} x^{4}\right) \mathrm{d} x .
$$

We use the method of layer-by-layer integration of (10) with respect to $\rho_{\mathbf{k}}$ suggested in $[1,8]$. The integration begins from the variables $\rho_{\mathbf{k}}$ with large values of $k$ (of the order of the Brillouin half-zone boundary) and terminates at $\rho_{\mathbf{k}}$ with $k \rightarrow 0$. For this purpose, we divide the phase space of the $\mathrm{CV} \rho_{\mathbf{k}}$ into layers with the division parameter $s$. In each $n$-th layer (corresponding to the set of wave vectors ${ }^{1}$$$
\mathcal{B}_{n}=\left\{\mathbf{k}=\left(k_{x}, k_{y}, k_{z}\right) \mid k_{i}=-\frac{\pi}{c_{n}}+\frac{2 \pi}{c_{n}} \frac{\kappa_{i}^{(n)}}{N_{i}^{(n)}} ; \kappa_{i}^{(n)}=1,2, \ldots, N_{i}^{(n)}\right\},
$$

where $N_{n}=N_{x}^{(n)} N_{y}^{(n)} N_{z}^{(n)}, N_{n}=s^{-3 n} N^{\prime}$, and $c_{n}=c^{\prime} s^{n}$. 
$\left.\mathbf{k} \in \mathcal{B}_{n} \backslash \mathcal{B}_{n+1}\right)$, the Fourier transform of the potential $\tilde{\Phi}(k)$ is replaced by its average value (arithmetic mean in the given case). To simplify the presentation, we assume that the correction for the potential averaging is zero, although it can be taken into account if necessary [1]. As the result of step-by-step calculation of partition function, the number of integration variables in the expression for this quantity decreases gradually. The partition function is then represented as a product of partial partition functions $Q_{n}$ of separate layers and the integral of the "smoothed" effective measure density $\mathcal{W}_{4}^{(n+1)}(\rho)[1,6]$ :

$$
Z=C_{n} Q_{0} Q_{1} \cdots Q_{n}\left[Q\left(P_{n}\right)\right]^{N_{n+1}} \int \mathcal{W}_{4}^{(n+1)}(\rho)(\mathrm{d} \rho)^{N_{n+1}}
$$

Here $C_{n}=2^{N} 2^{\left(N_{n}-1\right) / 2} \mathrm{e}^{\widetilde{\mathcal{M}} 0 N}$. The quartic measure density of the $(n+1)$-th block structure $\mathcal{W}_{4}^{(n+1)}(\rho)$ has the form

$$
\begin{aligned}
\mathcal{W}_{4}^{(n+1)}(\rho)= & \exp \left[-\frac{1}{2} \sum_{\mathbf{k} \in \mathcal{B}_{n+1}} d_{n+1}(k) \rho_{\mathbf{k}} \rho_{-\mathbf{k}}\right. \\
& \left.-\frac{a_{4}^{(n+1)}}{4 ! N_{n+1}} \sum_{\substack{\mathbf{k}_{1}, \ldots, \mathbf{k}_{4} \\
\mathbf{k}_{i} \in \mathcal{B}_{n+1}}} \rho_{\mathbf{k}_{1}} \cdots \rho_{\mathbf{k}_{4}} \delta_{\mathbf{k}_{1}+\cdots+\mathbf{k}_{4}}\right]
\end{aligned}
$$

where $d_{n+1}(k)=a_{2}^{(n+1)}-\beta \tilde{\Phi}(k)$ and $a_{2 l}^{(n+1)}$ are renormalized values of the coefficients $a_{2 l}^{\prime}$ after integration over $n+1$ layers of the CV space. The corresponding renormalization group transformation can be related to the Wilson type [9]. The main feature is the integration of short-wave modes, which is generally done without using Gaussian perturbation theory. The short-wave modes are described by a non-Gaussian measure density.

We found explicit expressions for the recurrence relations arising after the stepby-step integration of the partition function over layers of the CV phase space at $h=0$ and connecting the coefficients of the measure densities of the $(n+1)$-th and $n$-th block structures (see [6] ). Expansions obtained for these relations contain powers of a small parameter $s^{-d}$. The found optimal parameter of division of the CV phase space on layers $s=s^{*}=3.4252$ is used in the subsequent calculations. The obtained solutions of recurrence relations

$$
\begin{aligned}
& r_{n}=r^{*}+c_{1} E_{1}^{n}+c_{2} R E_{2}^{n}, \\
& u_{n}=u^{*}+c_{1} R_{1} E_{1}^{n}+c_{2} E_{2}^{n}
\end{aligned}
$$

are used to calculate the free energy of the system. Here $r_{n}=s^{2 n} d_{n}(0), u_{n}=s^{4 n} a_{4}^{(n)}$. The quantities $r^{*}=-f_{0} \beta \tilde{\Phi}(0)$ and $u^{*}=\varphi_{0}(\beta \tilde{\Phi}(0))^{2}$ are fixed point coordinates. The coefficients $c_{l}$ are written as

$$
\begin{aligned}
& c_{1}=\left[a_{2}^{\prime}-\beta \tilde{\Phi}(0)-r^{*}-\left(a_{4}^{\prime}-u^{*}\right) R\right] D^{-1}, \\
& c_{2}=\left[a_{4}^{\prime}-u^{*}-\left(a_{2}^{\prime}-\beta \tilde{\Phi}(0)-r^{*}\right) R_{1}\right] D^{-1},
\end{aligned}
$$


where $R=R^{(0)}\left(u^{*}\right)^{-1 / 2}, R_{1}=R_{1}^{(0)}\left(u^{*}\right)^{1 / 2}$. The temperature-independent quantities $R^{(0)}=R_{12}^{(0)}\left(E_{2}-R_{11}\right)^{-1}, R_{1}^{(0)}=\left(E_{1}-R_{11}\right)\left(R_{12}^{(0)}\right)^{-1}$ and $D=\left(E_{1}-E_{2}\right)\left(R_{11}-E_{2}\right)^{-1}$ are determined by elements $R_{12}^{(0)}, R_{11}$ and eigenvalues $E_{l}\left(E_{1}>1, E_{2}<1\right)$ of the renormalization group linear transformation matrix.

Calculating separately the contributions to the free energy from the short-wave $\left(F_{\mathrm{CR}}\right)$ and long-wave modes of the spin moment density oscillations both above and below critical temperature $T_{\mathrm{c}}\left(F_{\mathrm{LGR}}\right.$ at $T>T_{\mathrm{c}}$ or $F_{\mathrm{IGR}}$ at $\left.T<T_{\mathrm{c}}\right)$, we can find a complete expression for the free energy of the system

$$
F=F_{0}+F_{\mathrm{CR}}+F_{\mathrm{LGR}(\mathrm{LGR})},
$$

the entropy $S=-\partial F / \partial T$, internal energy $U=F+T S$ and specific heat $C=$ $T \partial S / \partial T$. Here $F_{0}=-k T N \ln 2$ is the free energy of $N$ noninteracting spins. The short-wave modes are characterized by a renormalization group symmetry. They correspond to the region of critical regime (CR) observed above as well as below $T_{\mathrm{c}}$. The renormalization group method is used here. The calculation of the expression describing the contribution from short-wave modes to the free energy involves the summation of partial free energies over the layers of the phase space of the CV up to the point at which the system leaves the CR region. In this case, it is important to obtain an explicit dependence on the number of the layer. For this purpose the solutions of recurrence relations are used. The short-wave modes facilitating the system instability are responsible for the formation of critical exponents and for the renormalization of the coefficient of the distribution describing the long-wave modes. The region of the limiting Gaussian regime (LGR) for $T>T_{\mathrm{c}}$ or the region of the inverse Gaussian regime (IGR) for $T<T_{\mathrm{c}}$ is associated with long-wave modes. The way in which the contribution from long-wave modes to free energy of the system is taken into account differs qualitatively from the method of calculating the shortwave part of the partition function. The calculation of this contribution is based on using the Gaussian measure density as the base density. We have developed a direct method of calculations with the results obtained by taking into account the short-wave modes as initial data. The regular temperature variation of the entropy and the specific heat positiveness are ensured by the contribution of the long-wave oscillation modes playing the role of a stabilizing factor. The major results obtained within the framework of the CV method are presented in $[1,5,8]$.

A distinctive feature of recurrence relations solutions (17) is the specific dependence of one of their coefficients $c_{1}$ on temperature $\left(c_{1}=\tau \tilde{c}_{1}\right)[1,6,8]$. Using the expression for $c_{1}(18)$ and the condition $c_{1}\left(T_{\mathrm{c}}\right)=0$ (then the solutions of recurrence relations in the CR region $r_{n}$ and $u_{n}$ with the increasing number of the layer $n$ tend to their values at a fixed point $r^{*}$ and $\left.u^{*}\right)$, we obtain an equation for $\beta_{\mathrm{c}} \tilde{\Phi}(0)$, which determines the phase transition temperature $T_{\mathrm{c}}[6]$ :

$$
\begin{aligned}
A_{\mathrm{c}}^{\prime}\left(\beta_{\mathrm{c}} \tilde{\Phi}(0)\right)^{2} & +B_{\mathrm{c}}^{\prime} \beta_{\mathrm{c}} \tilde{\Phi}(0)+D_{\mathrm{c}}^{\prime}=0 \\
A_{\mathrm{c}}^{\prime} & =1-f_{0}-R^{(0)} \sqrt{\varphi_{0}} \\
B_{\mathrm{c}}^{\prime} & =-a_{20} \\
D_{\mathrm{c}}^{\prime} & =a_{40} R^{(0)} \varphi_{0}^{-1 / 2}
\end{aligned}
$$


where $a_{20}=a_{2}^{\prime}\left(T_{\mathrm{c}}\right), a_{40}=a_{4}^{\prime}\left(T_{\mathrm{c}}\right)$ (see (11)). The coefficients $B_{\mathrm{c}}^{\prime}$ and $D_{\mathrm{c}}^{\prime}$ are functions of temperature $\beta_{\mathrm{c}} \tilde{\Phi}(0)$. They also depend on the interaction potential range $b$ and on the quantity $\bar{\Phi}$. The solution of the equation (20) is obtained by taking into account the dependence of its coefficients on $\beta_{\mathrm{c}} \tilde{\Phi}(0)$. Results of the calculations are presented in figure 1. It should be noted that the behaviour of the inverse phase

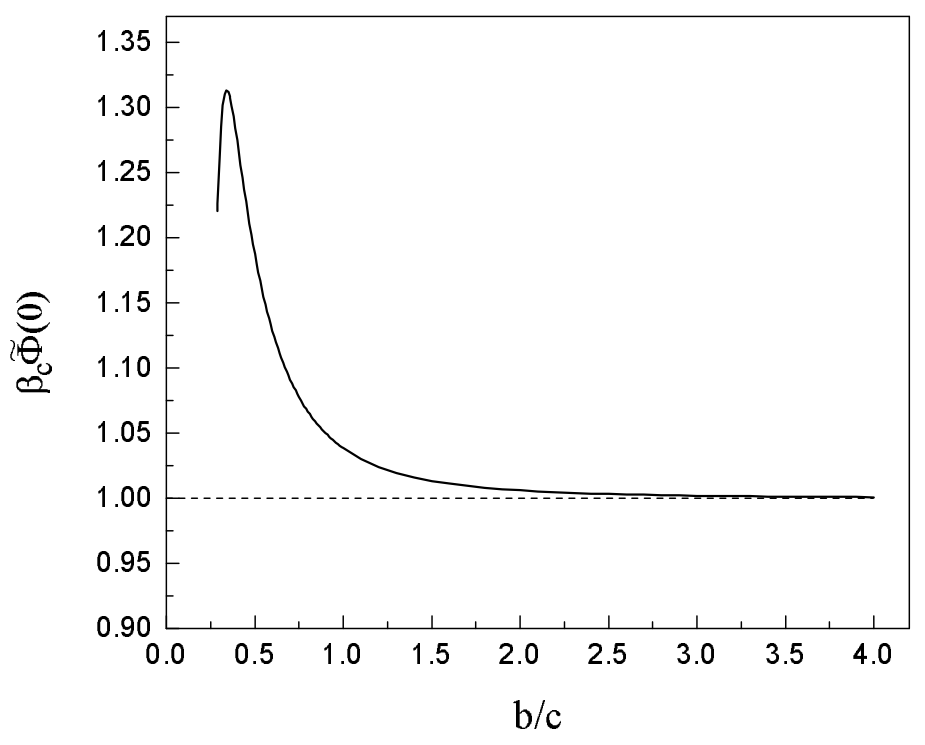

Figure 1. Dependence of $\beta_{\mathrm{c}} \tilde{\Phi}(0)$ on the ratio of the interaction potential range $b$ to the lattice constant $c$.

transition temperature with an increasing potential range is also in agreement with the data for the three-dimensional spherical model [10].

For the nearest neighbour interaction $\left(b=b_{I}, \tilde{\Phi}(0)=2 d J\right)$ we have $\beta_{c} \tilde{\Phi}(0)=$ 1.221 or $\beta_{\mathrm{c}} J=0.203$. Our result for $\beta_{\mathrm{c}} J$ accords with the results obtained by other methods, for example, with the value $\left(\beta_{\mathrm{c}} J\right)^{-1}=4.5103$ (or $\beta_{\mathrm{c}} J=0.2217$ ) $[11,12]$ calculated using the high-temperature series data. The similar value is also obtained using the real space renormalization group method based on the cumulant expansion $\left(\beta_{\mathrm{c}} J=0.22401[13]\right)$ and the Monte Carlo method $\left(\beta_{\mathrm{c}} J=0.221654 \pm 0.000006\right.$ [14], $\left.\beta_{\mathrm{c}} J=0.2216595 \pm 0.0000026[15-17]\right)$. The dependence of the inverse phase transition temperature $\beta_{\mathrm{c}} A=\beta_{\mathrm{c}} \tilde{\Phi}(0) /\left[8 \pi(b / c)^{3}\right]$ in units of $A$ ( $A$ is a constant entering the interaction potential $(2))$ on the ratio of the microscopic parameters $b$ and $c$ is shown in figure 2.

Let us mention that the proposed method of calculation permits to find not only the phase transition temperature but also the thermodynamic characteristics of the system as functions of the microscopic parameters (the lattice constant and parameters of the interaction potential). There is a possibility to investigate the dependence of various physical quantities near $T_{\mathrm{c}}$ on the microscopic parameters of the initial system that makes this method useful in describing the phase transitions in a wide class of three-dimensional systems. The methods existing at present make it possible to calculate critical characteristics to a quite high degree of accuracy 


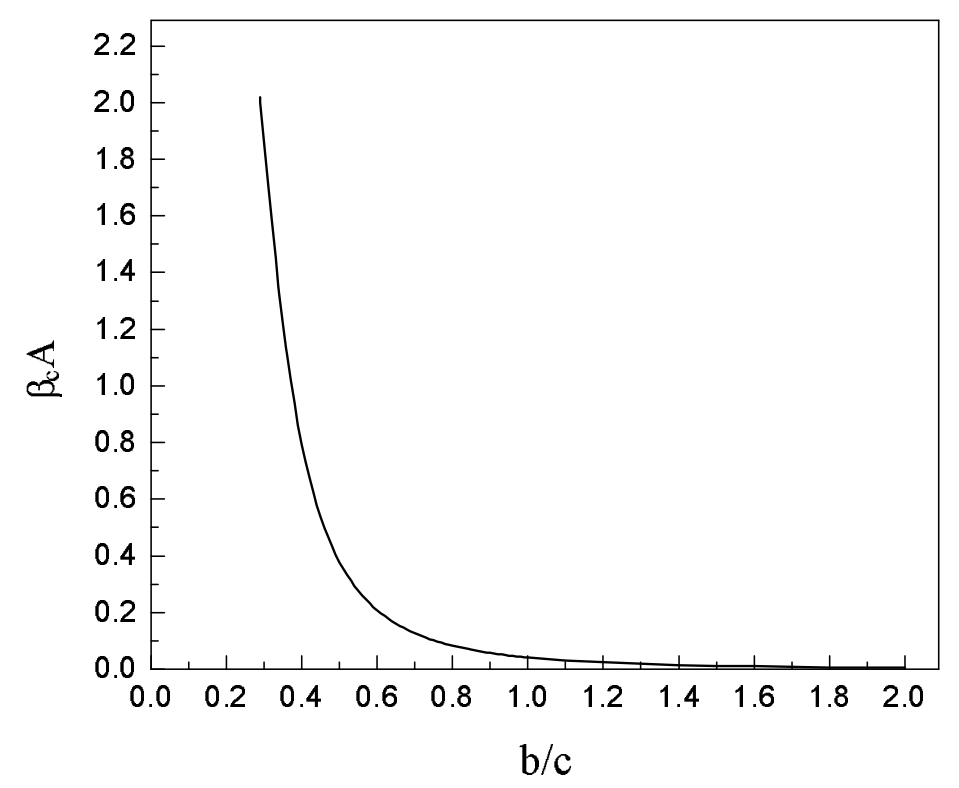

Figure 2. Critical temperature $\beta_{\mathrm{c}} A$ as a function of the microscopic parameters of the system.

(see, for example $[17,18])$. The advantage of the method under investigation lies in the possibility to obtain and analyze expressions for thermodynamic characteristics and their amplitudes as functions of microscopic parameters of the system [2-5, 19]. It is shown (see, for example, $[2,4,20,21]$ ) that the leading critical amplitudes and the correction-to-scaling amplitudes for the specific heat, average spin moment, susceptibility of the system can be presented in the form of the product of the universal part, independent of microscopic parameters, and the nonuniversal factor, which depends on these parameters.

\section{References}

1. Yukhnovskii I.R. Phase Transitions of the Second Order. Collective Variables Method. Singapore, World Scientific, 1987.

2. Kozlovskii M.P., Pylyuk I.V., Yukhnovskii I.R. Thermodynamic functions of threedimensional Ising model near the phase transition point with allowance for corrections to scaling. I. The case $T>T_{\mathrm{c}}$. // Theor. Math. Phys., 1991, vol. 87, No. 2, p. 540-556.

3. Yukhnovskii I.R., Kozlovskii M.P., Pylyuk I.V. A method for the calculation of thermodynamic functions for the 3D model systems in the critical region. // Z. Naturforsch., 1991, vol. 46a, p. 1-7.

4. Kozlovskii M.P., Pylyuk I.V. Entropy and specific heat of the 3D Ising model as functions of temperature and microscopic parameters of the system. // Phys. Stat. Sol. (b), 1994, vol. 183, p. 243-249.

5. Pylyuk I.V. Critical behavior of the three-dimensional Ising system: Dependence of thermodynamic characteristics on microscopic parameters. // Theor. Math. Phys., 1998, vol. 117, No. 3, p. 1459-1482. 
6. Kozlovskii M.P., Yukhnovskii I.R. Method for the self-consistent description of the critical behaviour of the three-dimensional Ising-like systems at a microscopic level. Preprint of the Institute for Condensed Matter Physics, ICMP-97-25U, Lviv, 1997, 96 p. (in Ukrainian).

7. Kozlovskii M.P., Pylyuk I.V., Usatenko Z.E. Method of calculating the critical temperature of three-dimensional Ising-like system using the non-Gaussian distribution. // Phys. Stat. Sol. (b), 1996, vol. 197, p. 465-477.

8. Yukhnovs'kii I.R. Solution of the three-dimensional Ising model for description of the second-order phase transition. // Riv. Nuovo Cim., 1989, vol. 12, No. 1, p. 1-119.

9. Wilson K., Kogut J. The Renormalization Group and the $\epsilon$-Expansion. Amsterdam, North-Holland, 1974.

10. Baker G.A., Jr. Certain general order-disorder models in the limit of long-range interactions. // Phys. Rev., 1962, vol. 126, No. 6, p. 2071-2078.

11. Moore M.A., Jasnov D., Wortis M. Spin-spin correlation function of the threedimensional Ising ferromagnet above the Curie temperature. // Phys. Rev. Lett., 1969, vol. 22, No. 18, p. 940-943.

12. Ferer M., Wortis M. High-temperature series and critical amplitudes for the spin-spin correlations of the three-dimensional Ising ferromagnet. // Phys. Rev. B, 1972, vol. 6, No. 9, p. 3426-3444.

13. Reich W. Determination of critical temperature for three-dimensional Ising systems. // J. Phys. A, 1984, vol. 17, No. 18, p. 3553-3558.

14. Pawley G.S., Swendsen R.H., Wallace D.J., Wilson K.G. Monte Carlo renormalizationgroup calculations of critical behavior in the simple-cubic Ising model. // Phys. Rev. B, 1984, vol. 29, No. 7, p. 4030-4040.

15. Ferrenberg A.M., Landau D.P. Critical behavior of the three-dimensional Ising model: a high-resolution Monte Carlo study. // Phys. Rev. B, 1991, vol. 44, No. 10, p. 50815091.

16. Landau D.P., Ferrenberg M.A. A high resolution Monte Carlo study of critical behavior: the three-dimensional Ising model. // J. Kor. Phys. Soc., 1993, vol. 26, p. S371S377.

17. Landau D.P. Computer simulation studies of critical phenomena. // Physica A, 1994, vol. 205, p. 41-64.

18. Liu A.J., Fisher M.E. The three-dimensional Ising model revisited numerically. // Physica A, 1989, vol. 156, No. 1, p. 35-76.

19. Kozlovskii M.P., Pylyuk I.V., Dukhovii V.V. Calculation method for the threedimensional Ising ferromagnet thermodynamics within the frames of $\rho^{6}$ model. // Cond. Matt. Phys. (Lviv), 1997, No. 11, p. 17-49.

20. Pylyuk I.V. Description of critical behavior of Ising ferromagnet in the $\rho^{6}$ model approximation taking into account confluent correction. I. Region above the phase transition point. // Low Temp. Phys., 1999, vol. 25, No. 11, p. 877-888.

21. Pylyuk I.V. Description of critical behavior of Ising ferromagnet in the $\rho^{6}$ model approximation taking into account the confluent correction. II. Region below the phase transition point. // Low Temp. Phys., 1999, vol. 25, No. 12, p. 953-961. 


\section{Залежність критичної температури від мікроскопічних параметрів у тривимірних ізінгоподібних системах}

\section{М.П.Козловський, В.В.Духовий, І.В.Пилюк}

Інститут фізики конденсованих систем НАН України, 79011 Львів, вул. Свєнціцького, 1

Отримано 21 березня 2000 р., в остаточному вигляді 5 листопада 2000 p.

В рамках методу колективних змінних досліджується критична поведінка тривимірної ізінгоподібної системи на простій кубічній гратці з експонентно спадним потенціалом взаємодії. Розв'язок рівняння для температури фазового переходу одержано в результаті врахування залежності його коефіцієнтів від температури. Досліджено залежність критичної температури від радіуса дії потенціалу взаємодії.

Ключові слова: ізінгоподібні системи, критична поведінка, фазові переходи, колективні змінні, температура фазового переходу

PACS: $05.50 .+q, 64.60 . F r, 75.10 . H k$ 